\title{
IL MERCATO NELL'ITALIA MEDIEVALE
}

Giovanni Cherubini

Università degli studi di Firenze

II Medioevo si apre, in Italia, con la crisi del sistema economico imperiale. Alla decadenza delle città e alla rovina dei ceti medi si acompagna, com'è noto, una crescente ruralizzazione della vita economica, demografica, sociale, e un complessivo sposamento di equilibri a favore delle campagne e della grande proprietà e un concomitante declino proggressivo della vita mercantile e del mercato. Questi processi non furono accellerati, soprattutto con l'arrivo, poco dopo la metà del VI secolo, dei Longobardi che, insediandosi in Italia, non riuscirono tuttavia a conquistarla completamente, ne determinarono per la prima volta dopo molti secoli dall'unificazioni realizzata da Roma una divisione destinata a durare ancora più a lungo. II declino della vita urbana e di tutto ciò che essa significava dal punto di vista economico ebbe anzi in Italia, rispetto al resto dell'Occidente, una propria specifità nella morte di molte vecchie e gloriose città, come Sibari, come Metaponto, come Roselle, come Populonia, come Vetulonia, come Luni, come Spina, come tantissime altre, nel corso di una lunga agonia durata sin nel pieno Medioevo e col passaggio delle loro funzioni, in più di un caso e prima fra tutte quella episcopale, ad altre e nuove città.

Con la decadenza del mondo urbano e la ruralizzazione della vita economica si acompagnò il declino progressivo di quello che era uno degli strumenti centrali degli scambi, vale a dire il magnifico sistema viario romano. Per quanto nato soprattutto con funzioni militari e di unificazione amministrativa, esso constituiva naturalmente una delle condizioni essenziali degli scambi. D'altra parte, la frantumazione dell'imperio nei regni romano-barbarici si ripercosse negativamente sul fitto e tradizionale reticolo di comunicazioni marittine che avevano al loro centro l'Italia. La stessa vicenda dell'espansione musulmana, senza voler qui riprendere l'annosa questione "Maometto-Carlomagno", determinò senza alcun dubbio, almeno temporalmente, una qualche difficoltà per la tranquillità, la continuità e la regolarità degli scambi. Ciò che si sa delle incursioni, delle distruzioni e poi degli insediamenti musulmani in Campania o Calabria rivela un arretramento di popolazioni verso l'interno e una chiusura verso il 
contatti esterni. Ma ciò che anche sappiamo di quel che avvenne un po'più tardi, almeno per qualche località come Amalfi o Gaeta, mostra che ai conflitti e agli scontri potevano alternarsi o succedere i contatti e gli scambi.

E' tuttavia su altre specifità della peninsola italiana che mi piace richiamare l'attenzione. Intanto, non ostante quella più o meno lenta morte di molti centri abitati sopra richiamata, la vita urbana pur nella contrazione fisica e demografica di tutte le città, mantenne una vivacità e un ruolo sconosciuti altrove in Occidente, esclusa soltanto la Spagna musulmana. $\mathrm{Ma}$ al mondo musulmano, del resto, una parte d'Italia, ciò̀ la Sicilia, e qualque altra piccola porzione cominciò ad appartenere allinizio del IX secolo. Ma la specifità italiana storicamente più rilevante è piuttosto un'altra, vale a dire la presenza di alcuni territori non sottomessi dai Longobardi e formalmente dipendenti da Bisanzio, poi lentamente diventati sostanzialmente indipendenti. In alcuni di questi territori nacquero e si svilupparono nuovi centri urbani, come Venezia e Amalfi, che costituirono nuovi precoci poli di sviluppo mercantile. La varia situazione della penisola conosce anche vicende come quella di Gaeta, che appare come collocata a mezza strada tra Roma e Bisanzio. Ma più che i contatti mercantili tra tutte queste città e Bisanzio, mi pare indispensable ricordare i contatti col mondo musulmano. Essi dimostrano, in pieno Medioevo, come alla stagione degli scontri e dell'incomunicabilità -se realmente questa incomunicabilità ci fu- fosse seguita l'età dei contatti. Si conosce, in particolare, la presenza, decisamente cospicua, di mercanti amalfitani in Egitto, nel corso del $\mathrm{X}$ secolo; si osserva che, a dispetto di tutte le ingiunzioni papali, questi mercanti dovevano esportare dalle montagne campane fra i musulmani anche prodotti di valore strategico come il legname.

"Forse già nel secolo VIII, e certamente nel secolo IX, i Veneziani, scrive Giono Luzzatto, hanno rapporti commerciali con la Sicilia, con la Grecia e con l'Egitto e si spingono talvolta essi stessi in quei paesi. Al principio dell' 800 li vediamo in possesso di una flotta da guerra con cui vanno in aiuto dei Greci: nello stesso tempo li incontriamo a Cremona ed a Pavia, come concorrenti dei mercanti di Comachio, a vendervi, assieme al sale, penne e pelli pregiate, velluti, sete, stoffe purpuree di Tiro: tutti prodotti orientali ch'essi scambiavano con legname, con ferro e soprattutto con schiavi"

Più che questi ben noti movimenti di Veneziani e di Amalfitani, più tardi seguiti da Pisani e da Genovesi, aperti verso l'esterno, un cenno merita il commercio nel regno longobardo. Almeno per la metà dell'VIII secolo si parla di un ceto di negotiatores tenuto in notevole considerazione e spartito in maiores o potentes e in sequentes e mibores. I maiores erano collocati al livello dei propietari di almeno sette poderi (case massaricie). Abbastanza numerosi compaiono nei documenti, accanto ai mercanti, gli artiginai residenti in città. 
$E$ ' da ricordare anche il ruolo dell'economia di scambio delle curtes del regno italico tra la fine dell'VIII e l'inizio dell'XI secolo, proprio perché si ritenne alla fine del secolo scorso che esistesse un "sistema curtense" indicatore di una fase economica specifica, quella dell'economia "naturale" o "domestica" o "del consumo interno", da contrapporre all'economia "monetaria". II predominio della grande proprietà, laica o eclesiastica, la tendenza autarchica conseguente alla diffusione del sistema organizzatore di questa proprietà, la rarefazione della moneta e la riduzione delle attività di scambio all'esterno delle entità patrimoniali sarebbero stati i caratteri salienti di quella fase storica. Nessuno può negare che in queste opinioni ci sia molto di vero, ma già molto presto ne venne respinta, almeno per l'Italia, la schematicità. Di recente, tornando sull'argomento, Pierre Toubert ha cercato di chiarire quale "fu il ruolo della moneta all'interno del sistema curtense" e di "valutare l'importanza della grande proprietà all'interno dell'economia globale; cioè, più precisamente, di accertare la sua funzione organizzativa non solo nei confronti della produzione agricola, ma anche degli spazi e delle reti dello scambio interno di cui peraltro la documentazioni attesta la vitalità". Le curtes, indipendentemente dalla loro costante bipartizione in pars dominica e terre concesse differivano profondamente l'una dell'altra per la differente destinazione delle terre (incolti, pascoli, cereali, colture specializzate). "Al primo livello di osservazione, quello dell'unità curtense, il sistema funziona già come sistema di trasferimento di servizi, di beni e di prodotti delle aziende contadine verso il centro di gestione della curtis". Giornate di lavoro sulla pars dominica, censi in natura e/o danaro, fornitura di prodotti artigianali dell'artigianato domestico da parte dei contadini costituivano il complesso di questo trasferimento. A partire dal secolo IX si parla anche di laboratori curtense, ciò̀ di luoghi dove il titolare della curtis faceva direttamente lavorare da dipendenti stoffe, tegole, oggeti di ceramica. Questi prodotti rimasero tuttavia, almeno in alcuno grandi complessi curtensi padani, meno importanti delle rimesse dell'artigianato domestico contadino. In questi grandi complessi, la curtis centrale giocava da polo di raccolta e di redistribuzione dei prodotti di tutte le curtes, che erano di regola molto differenziati. Valga per tutti il caso, del resto ben conosciuto, della olivicultura della regione dei laghi, oppure quello del miele e dei formaggi provenienti dalle curtes con orientamento silvo-pastorale. Questi prodotti vengono trasferiti a distanza, spesso per via d'acqua, utilizzando una parte della manodopera in corvées di trasporto. Mercati curtensi e mercati urbani, strettamente legati fra loro, sono i destinatari di queste produzioni. tutti il grandi proprietari fondiari -conosciamo per la verità, dato lo stato della documentazione, soltanto quelli monastici- hanno, allo scopo, delle succursali nelle città. "Sarebbe certamente esagerato trarre da tutto ciò la conclusione che i grandi proprietari eclesiastici siano riusciti allora a costruire intorno alle città una economia di mercato differenziata. $E^{\prime}$ vero che i padroni delle grandi proprietà fondiarie sono presenti sui mercati 
urbani; in questi mercati hanno maggiori probabilità di trovare i prodotti rari ed esotici o gli oggetti curiosi che lusingano il gusto e soddisfano il bisogno di distinzione sociale. Ma per il resto, cioè per l'essenziale, niente distingue ancora realmente le città dai mercati curtensi; né la natura dei prodotti di base, né il ritmo delle attività, né la qualità degli agenti economici". Nemmeno i prodotti rari "vengono tutti dall'Oriente". "La caratteristica più importante di questo commercio (...) rimane, nel secolo X, la struttura d'impronta ancora marcatamente patrimoniale delle reti organizzate dai grandi proprietari intorno ai mercati gerarchizzati in funzione delle necessità della gestione curtense".

Nei trasferimenti delle merci è presente lo strumento monetario, e del resto in moneta sono anche molti censi che i contadini devono versare al padrone della curtis. La riforma carolingia, susseguente alle svalutazioni del tremisse d'oro longobardo, avrebbe offerto i mezzi adatti di pagamento anche per i più bassi livelli economici. Tuttavia almeno tre considerazioni finali si impongono. Non sappiamo quale fosse la quota di produzione agraria e il valore dei prodotti manifatturati immessi sul mercato, ma non se ne deve esagerare l'importanza. Non sappiamo quale fosse in particolare lo spazio che come venditori o come compratori vi occupavano i contadini, ma tutto fa pensare che questo fosse modesto. Non possiamo tacere che i surplus venduti dai proprietari di curtes derivavano dal lavoro di una manodopera non libera.

Il periodo chiamato, per tutta l'Europa e tanto più per I'Italia, dell'espansione, inizia grosso modo a cavallo tra il X e I'XI secolo. Su di questo desidero richiamare più particolarmente l'attenzione. come data finale scelgo i primi deceni del XIV secolo. Tengo quindi fuori, per non complicare ulteriormente un quadro già abbastanza complesso, la crisi demografica e i suoi effetti sull'economia. L'esame di questi secoli di espansione è particolarmente interessante proprio perché, anche in relazione al mercato, appaiono evidenti e profonde novità, ma anche elementi di continuità e strozzature.

Che a partire dall'inizio dell'XI secolo o anche un po' prima gli scambi si siano in Italia $O$ anche dall'Italia verso l'esterno infittiti, intensificati e irrobustiti, nessuno dubita. Le prove sono, per la verità, di tipo qualitativo o addirittura induttivo, piuttosto che quantitative. Esse risultano però cosi convergenti, molteplici, varie che il fenomeno appare del tutto certo. Lo sviluppo degli scambi ha lasciato tracce persino nella toponomastica delle campagne con la nascita di molti luoghi abitati battezzaati mercatale o con espressioni simili.

II motore centrale di questa espansione degli scambi pare essere stato costituito dalle città. Nel periodo considerato esse crebbero molto vigorosamente sino a dar vita, nella parte centro-settentrionale della penisola, ad un reticolo urbano che non aveva uguali in Europa, per dimensione di alcuni centri maggiori, fittezza delle città, livello di urbanizzacione. Non ostante il fitto reticolo dei centri antichi, che ora 
riprendevano vita, no mancò neppure -certo in misura infinitamente meno cospicua che in altre regioni europee -la nascita di nuovi abitati, con connotati o anche poi con posizione formale di città. Alle origini il ruolo maggiore di questa ripresa dei commerci pare essere stata rappresentata sopratutto dalle città marittime di Venezia, Genova, Pisa (Amalfi patisce già nel XII secolo l'inizio di una crisi decisiva, forse anche come conseguenza della conquista normanna). Le crociate dettero, com'è noto, un forte impulso allo sviluppo commerciale di queste città, per la concessioni che esse ottenero in conseguenza della loro partecipazione a quelle imprese. La loro affermazione, militare oltre che commerciale, era cominciata tuttavia un po' prima, da parte di Venezia sulle coste dalmate, da parte di Pisa e Genova nelle imprese contro vari caposaldi musulmani nelle acque tirreniche.

Dopo le città marittime l'intensificazione degli scambi toccò anche le città dell'interno. Di alcune si può sottolineare il ruolo avuto in questo sviluppo dalla presenza di qualche importante via di comunicazione: così per Ferrara fu importante il controllo -poi contestato da Venezia- dei vari bracci fluviali del delta del Po; cossi per Piacenza il trovarsi essa all'incrocio tra la via rappresentata dal grande fiume e la via terrestre della Francigena che dalla Manica conduceva verso Roma; cosi per Firenze, forse, la conremporanea possibilità di utilizzare l'Arno e una serie di vie terrestri; così per Siena il trovarsi sulla Francigena (dalla quale trassero, come sembra, origine, e se non proprio origine, almeno fortuna e sviluppo anche centri più modesti come San Gimignano o Colle Valdelsa). In certi casi, come quello di Milano, il discorso sembra più complesso, potendosi tirare in campo più motivi, dal sistema vario alla ricchezza delle produzioni locali.

Non è mia intenzione tracciare di nuovo in questa sede i caratteri e i confini di quella sorta di impero commerciale che i mercanti italiani avevano messo in piedi tra la fine del XIII e l'inizio del XIV secolo. Esso andava da Bisanzio alla Spagna, dalle coste africane all'Irlanda e all'Inghilterra, con caposaldi anche in Germania e nell'Europa centrale, ma generalmente con un confine segnato dalla presenza degli interessi anseatici. Basti qui precisare che quell'impero, cui partecipavano in misura diversa molte altre città, anche piccole, aveva i suoi punti di forza sopratutto in Venezia, Genova e Firenze (con una qualche minore importanza, almeno a quella data finale, di Milano e della declinante Pisa).

$\mathrm{Mi}$ pare opportuno soffermanrmi ivence sul livello e sui caratteri degli scambi all'interno della penisola italiana. In questa agivano prepotentemente, per qual che riguarda le produzioni e la loro circolazione, due tendenze opposte. Una era orientata verso la libera circolazione dei beni, o almeno di alcuni beni, anche al di là delle molte barriere politiche rappresentate dai piccolo stati cittadini; l'altra tendenza, al contrario, mirava, in ognuno di quegli stati, a dar vita ad un sistema economico autarchico, sia per quanto riguarda la produzione di beni agricole sia per 
quanto riguarda la produzione di beni artigianali. Le due tendenze coesistevano in ciascuna città, così che, mentre si aveva la tendenza ad esportare prodotti finiti, si cercava, con una serie di dazi o accorgimenti, di frenare l'afflusso di prodotti provenienti da altre città. E d'altra parte, al contrario, proprio le città di pi più forte struttura produttiva, che erano anche spesso le più popolose, si sforzavano di attirare dall'esterno le derrate alimentari per nutrire i loro abitanti. Le contrapposte tendenze non impedirono, attraverso accordi tra città, la differenziazione delle produzioni, la specifità di certe produzioni per certe città e lo sviluppo degli scambi anche all'interno del paese. Ma giova precisare che la barriere politiche di tanti stati cittadini, la compresenza di residui feudali indipendenti in certi aree marginali, l'esistenza di regni nel Mezzogiorno e nelle isole, non avevano dato vita, all'inizio del Trecento, non ostante tutte le novità, ad un vero e proprio mercato nazionale.

I circuiti del commercio italiano conoscevano dunque, con i limiti ora detti, un ambito relativo alla penisola e un ambito internazionale. C'era poi l'area di scambio rappresentata dalla città e dal territorio da essa dipendente, che comportava una netta gerarchia tra il mercato della città e quello dei centri maggiori o minori delle campagne. C'era infine, spesso, un'area più vasta nella quale una città maggiore, anche senza prima di imporre la sua dominazione politica, era riuscita ad imporre la sua supremazia economica. Il caso più cospicuo e più noto è quello di Venezia, che in larga parte riuscì a costruire una sorta di monopolio mercantile sulle terre romagnole, venete, marchigiane. $V a$ anche precisato che nei luoghi in cui lo scambio era, si può dire, giornaliero, meno rilievo avevano le fiere e i mercati stagionali. Nelle grandi città queste manifestazioni costituivano talvolta soprattutto un indispensabile corollario delle feste patronali, quando affluiva in città anche gente della campagna. Significativamente, nel regno del sud, meno segnato dagli scambi, era invece in piedi un vero sistema di fiere. Questo non esclude, naturalmente, l'esistenza di mercati settimanali, anche nei centri del territorio, per i più consueti bisogni locali; e neppure significa la mancanza di un mercato stabile, giornaliero, nelle città, della frutta, della cacciagione, del pollame, delle verdure, delle uova, e anche di qualque prodotto manifatturato, che si affiancava al mercato stabile delle botteghe. Questa presenza del mercato è anzi visivamente ben documentata, in molte città italiane, dalla sopravvivenza di logge al coperto per le erbe, per il pesce, per la carne. Si sbaglierebbe tuttavia a pensare che la città fosse schematicamente differenziata delle sue campagne, per la presenza, in quella, di uno scambio giornaliero e continuato dei beni, e per la presenza, nei centri della campagna, di scambi soltanto settimanali. In realtà la documentazione prova abbondantemente, almeno per il secolo XIII e per il primi decenni del XIV secolo, che molti centri abitati del territorio avevano le loro botteghe, i loro artigiani che producevano per la clientela di un determinato e ristretto raggio all'intorno, e anche veri e propri rivenditori di beni. Si può forse 
ipotizzare, con qualche ragione e in base a qualche esempio conosciuto, che il bottegario di paese avesse una bottega fornita di articoli più vari, anzi curiosamente diversi l'uno dall'altro, rispetto al rivenditore $e$ al commerciante della città.

In questa Italia fortemente segnata dai commerci esistevano naturalmente alcune più forti o piùdeboli direttive di traffico. Esistevano anche città con più spiccate connotazioni mercantili e città con più spiccate connotazioni manifaturiere. Fra le prime possiamo inserire le maggiori città marittime, tra le seconde soprattutto Milano, che era insieme un centro di produzione di stoffe e un centro di produzione di oggetti di ferro, armi in particolare. Firenze mostra invece una struttura economica ben equilibrata tra produzione e commercio, compreso naturalmente il comercio del denaro. Tra le maggiori direttive di traffico sono da indicare quella della Valpadana che faceva capo a Venezia, quella che dalla regione dei laghi conduceva a Genova e a Pisa, quella che da Firenze attraverso Bologna, Ferrara e Padova raggiungeva Venezia, quella che da Firenze scendeva lungo l'Arno sino al porto di Pisa, quella che percorreva la Francigena da Piacenza a Parma a Lucca a San Gimignano, a Siena, quella che da Arezzo e in genere dalla Toscana sfociava ad Ancona, quella che dalla Sicilia, ingrossata dal traffico campano e sardo, giungeva a Pisa e a Genova, quella che dalle Puglie raggiungeva Firenze, ed infine quella che dalle Puglie risalendo I'Adriatico metteva capo a Venezia.

Più discussi sono invece i volumi di quel commercio. La storiografia ha tuttavia ormai corretto l'idea troppo riduttiva che non aveva Werner Sombart. Pur riconoscendo che non si trattava di quantità paragonabili a quelle post-rivoluzione industriale, gli studiosi hanno ben dimostrato, come vedremo fra poco, che non viaggiavano soltanto beni di alto valore specifico, ma anche beni molto pesanti. Hanno anche dimostrato, ma sfortunatamente in caso troppo limitati e per gli anni finali del periodo qui considerato, che pesi fiscali, $m$ pedaggi e costi di trasporto costituivano percentuali modeste del costo finale della merce, dell'ordine rispettivamente dell' $1-2 \%$ e del $4-5 \%$.

Un ruolo importante negli scambi avevano le derrate agricole, non soltanto in ambito locale, e tra campagna e città, ma anche a distanza o grande distanza almeno per quello che riguarda i cereali, che erano l'ingrediente fondamentale dell'alimentazione. Circolava in grande quantità anche il sale. Circolava, anche a distanza, il bestiame. Fra i beni non destinati all'alimentazione sono da mettere al primo posto le lane, le pelli, le materie coloranti o mordenti come il guado, lo zafferano, l'allume. Fra i prodotti finiti, nello scambio a distanza, erano in primo luogo le stoffe, ma si commerciavano anche le armi. E' in definitiva completamente da respingere, per l'Italia di quei secoli di sviluppo, l'immagine di un traffico incentrato esclusivamente o quasi esclusivamente sulle spezie e i prodotti di valore. 
Tutto questo detto, si deve tuttavia precisare che lo scambio dei beni incontrava ostacoli molteplici, sia dal punto di vista tecnico, che giuridico, che politico, che stagionale. Le nevi sui monti e la stagione delle tempeste rallentavano o fermavano rispettivamente $\mathrm{i}$ trasporti per via di terra e quelli per via di mare. La primitività delle strade, in molti casi poco più che viottole e, data la conformazione geografica della penisola, percorribili soltanto a cavallo o a piedi, rendevano più convenienti i trasporti per mare e per fiume. Si ebbero così, nei secoli considerati, ad opera o per impulso dei governi cittadini, sensibili miglioramenti tecnici nel fondo stradale, apertura di valichi montani, costruzione di ponti, sviluppo di una fitta rete di alberghi. Furono aperti, nella pianura padana, canali non soltanto al fine di regolamentare le acque e bonificare le terre, ma anche per scopi commerciali, talvolta in colaborazione tra l'una e l'altra città, e tavolta in acerrima concorrenza per strapparsi correnti di traffico e relativi redditi. Nell'affermazione del potere cittadino sulle campagne e nella lotta contro i signori del territorio un aspetto rilevante fu la richiesta abolizione o riduzione del pedaggi. Tra città e città venne elaborato tutto un sistema di regolazione o eliminazione del primitivo sistema della rappresaglia. Nei trasporti marittimi venne elaborato uno specifico diritto della navigazione. I grandi mercanti con interessi e attività internazionali dettero vita, già entro la fine del Duecento, ad un sistema di posta che faceva circolare le notizie con relativa velocità rispetto alle abitudini di quel tempo.

Gli scambi venivano ostacolati, in mare da pirati e corsari, e soprattutto sulle vie di terra da un enemico grigantaggio. Non ostante la fittezza delle città, questo era presente, nei passi obbligati, nei luoghi boscosi, nella montagna, ai confini tra l'uno e l'altro stato, anche nelle regioni dell'Italia superiore, per quanto esso caratterizzasse, un po' come avvenne nel corso dell'età moderna, soprattutto il Lazio e le regioni meridionali. Contro questo pericolo, ma talvolta con scarso successo, i governi rafforzarono l'apparato repressivo, reseo le strade più praticabili, riuscirono talvolta ad organizzare un'azione comune. Contro i pericoli cui andavano incontro le merci si sviluppò, com'è ben noto, anche un sistema di assicurazioni marittime, cui seguirà più tardi un sistema di assicurazioni su terra. Al miglioramento tecnico dei trasporti contribuì la nascita di vere e proprie organizzazioni di vetturali, mulattieri, carradori, portatori. Al miglioramento economico dette un contributo la coniazione, a partire dalla metà del Duecento, di forti e stabili monete auree, poi universalmente accettate, ad opera di Genova, Firenze, Venezia, che portarono un po' d'ordine, almeno in campo internazionale e negli scambi all'ingrosso, nella confusione delle menete che tutte le città erano venute coniando per gli accresciuti bisogni commerciali.

Un più particolare sottolineatura meritano gli effetti dello sviluppo del mercato sulla condizione personale dei contadini, sulle strutture fondiarie, sul sistema coltorale. Per quanto il fenomeno sia riconducibile anche ad altre cause, e comunque alla spinta interna alla società rurale e 
alla politica delle città, nell'talia del Centro-Nord la riduzione degli spazi della signoria e la crescita della libertà condadina andarono di pari passo. Ma con la crescita della libertà crebbe la circolacione della terra, si sviluppò la stratificazione sociale e una grandissima parte dei contadini fu anzi espropiata dei propri possessi dagli abitanti delle città. I contadini espropiati riebbero come coltivatori liberi e con contratti pazziari a breve termine terre più estese e meglio organizzate daglo abitanti delle città. Su quelle terre si fecero sentire le richieste del mercato urbano non soltanto per i prodotti alimentari, ma anche con la domanda di prodotti per l'industria, come le materie tintorie.

Non vorremmo tuttavia che da quanto ora detto si concludesse che, per chi vi parla, I'Italia dei secoli XI-XIII era il paradiso del mercato. Esistevano in effetti molti elementi contradittori con quanto ora detto, che fanno apparire il paese, per questo come per molti altri aspetti della sua civiltà che in questa sede non ci interessano, come straordinariamente anticipatore rispetto a quello che avvenne più tardi in Europa, ma anche come ancora segnato di elementi tradizionali. Si era formato un vero mercato del lavoro, che trascendeva spesso gli stessi confini delle piccole patrie cittadine. Tuttavia, mentre per l'edilizia, o per il lanificio, o per il bracciantato agricolo, era generalizzato il sistema del salario, nei nuovi rapporti agrari di cui abbiamo detto, il compenso della famiglia contadina che riceveva un'azienda da coltivare era costituito da una quotaparte dell'annua produzione agricola. Ma persino nell'avanzato settore urbano del lanificio nella forma della "fabrica disseminata" organizzata dal mercante-imprenditore, sappiamo che i salari degli operai fissati in moneta venivano di frequente, almeno in parte, pagati in prodotti.

Cé da dire anche che non di tutti i beni c'era un libero scambio, o almeno uno scambio soltanto dal gioco della domanda e dell'aofferta. II sistema corporativo, vigente in molte produzioni e sicuramente nelle più importanti, con il suo divieto della concorrenza fra soci e con il suo obbligo di iscrizione all'arte per poter esercitare un mestiere, conduceva ad una sorta di monopolio collettivo da parte di una categoria di produttori e quindi ad un prezzo del prodotto nel quale influivano semmai teorie moralreligiose come quella del giusto prezzo, ma non certo il libero gioco della domande e dell'offerta. Passando al settore delle derrate alimentari va invece segnalato che la più importante, cioè i cereali, doveva sottostare agli orientamenti della politica annonaria dei governi -sulla quale ritorneremo- e a tutti i lacciuoli, prezzo politico della merce compreso, che da quella derivavano.

La situazione era varia anche sul piano geografico. Gli scambi erano più intensi nella valle padana, nella Toscana, nel Veneto e nell'Italia superiore in genere, ma meno intensi, anche in queste regioni, nelle aree montane e più marginali. C'è poi da osservare che in intere sub-regioni come la Sardegna interna o la montagna calabrese il baratto doveva avere ancora una qualche importanza. E c'è, più in generale, da aggiungere che 
gli scambi a distanza determinarono via via, a partire dall'XI secolo, una particolare gerarchia economica fra le diverse parti del paese. Quella centro-settentrionale, caratterizzata dalla presenza della città autonome, forniva prodotti finiti, servizi e capitali, e quella meridionale, o dei regni, forniva derrate alimentari e materie prime, come grani, olio, pesce salato, vini, bestiame, lana, pellami.

Per un quadro più completo gli scambi devono essere esaminati anche sotto la prospettiva del rapporto tra il centro urbano e le sue campagne. Il discorso vale, in primo luogo, per le città dell'Italia centrosettentrionale, che sulle campagne avevano una supremazia politica oltre che economica. In generale si può osservare che mentre la città riversava sul contado le sue produzioni industriali, il contado faceva affluire in città le sue produzione agricole. Lo faceva tuttavia in tre modi diversi: attraverso gli affitti o le quote in natura sui raccolti corrisposti dai contadini ai proprietari delle terre, attraverso l'obbligo fatto al contado, con la politica annonaria, di far affluire entro le mura urbane tutti i cereali avanzati dal consumo locale, infine attraverso la libera vendita di altri prodotti dei campi, dei prati o dei boschi. Questo schema comportava, naturalmente, da un luogo all'altro, qualche eccezione o presentava almeno qualche sfumatura. In certi abitati più cospicui del territorio si continuava a produrre qualche manufatto, comprese le stoffe, magari meno pregiato o diverso da quelli prodotti in città. Le famiglie contadini continuavano a filare e a tessere qualche pezza di panno per il proprio autoconsumo. Su qualche distretto rurale la città non era riuscita ad imporre il proprio completo dominio, e quindi si manteneva, anche per questo aspetto, una qualche autonoma vitalità.

Ma un punto di particolare importanza, nella prospettiva di queste pagine, riguarda la misura della partecipazione dei contadini al mercato. Non abbiamo per questo aspetto dati quantitativi, né sono state tentate, con questo intento, ricerche specifiche. Le mie considerazioni si basano, conseguentemente, soltanto su una certa personale conoscenza di quella società $\mathrm{o}$ comunque su dati puramente indicativi. Intanto, anche per questo aspetto, poco o nulla sappiamo per il Mezzogiorno, salvo le cose generalissime dette poco più sopra. Per le campagne dell'Italia superiore possiamo osservare che raramente e soltanto, forse, in qualche area particolare e in certi anni i contadini vendevano sul mercato, con regolarità, la propria produzione cerealicola. Essa serviva infatti, in primo luogo, per l'alimentazione e l'autoriproduzione fisica della famiglia contadina, funzione parzialmente esplicata in qualche particolare distretto anche dalle castagne. Era semmai ad altri prodotti che il lavoratore dei campi chiedeva qualche ricavato monetario. Neppure il vino, che pur era il secondo prodotto per importanza, costituiva, di regola, un prodotto per la vendita, ma semmai un prodotto per mettere in pari i conti col padrone della terra, dal momento che quei conti erano sempre in deficit per il contadino. Intensa era invece la vendita sul mercato, sia direttamente in città, sia nei 
mercati del territorio, comunque entro una distanza percorribile nei due sensi in una sola giornata, del prodotti del pollaio -animali e uova-, della legna da ardere, della verdura e della frutta di stagione, del latte, di qualque modesto lavoro artigianale, come canestri o cappelli di paglia. Si creava dunque una specie di doppio circuito commerciale, nel quale ad immettere sul mercato i beni di base come cereali e vino erano soprattutto i propietari, particolarmente i maggiori, ma non i lavoratori della terra. $\mathrm{Ma}$ c'è da aggiungere che il contadino era in parte sottatto allo scambio monetario anche perché certi pavori che lui, la moglie o le figlie fornivano ai cittadini, come l'attività di balia, di serva domestica, di filatrice a domicilio, venivano molto spesso compensati non in moneta, ma attraverso il pagamento, da parte dei cittadini, presso i fornitori, delle calze, degli abiti, delle medicine o di altro che essi vi avevano comprato. Quando il cittadino, che con il contadino si trovava in contatto per i motivi sopra esposti, era un commerciante in stoffe, il regolamento di questi conteggi avveniva, per così dire, con un meccanismo del tutto interno al loro rapporto. Ma non si deve tuttavia esagerare, neppure per quelle aree del Mezzogiorno che abbiamo sopra rilevato come più arretrate. Si può infatti ragionevolmente supporre che se non i cereali -e per le ragioni dette-, almeno quelle grandi quantità di nocciole, quel vino, quell'olio pugliese da destinare soprattutto alla produzione di sapone e al trattamento delle lane, quegli svariati prodotti che i grandi uomini d'affari del Centro-Nord raccoglievano nei porti del Sud, spesso attraverso l'intermediazione di minori commercianti e accaparratori locali, provenissero in parte, e forse in grande parte, da una miriade di piccole economie contadine.

Questa Italia sviluppata, tutta percorsa da correnti intense di traffico e tutta punteggiata di luoghi di mercato, coinvolse, in definitiva, nelle attività di scambio, sia pure in misura molto diversa, tutti i ceti sociali.

\section{BIBLIOGRAFIA ESSENZIALE}

G. LUZZATO, Storia economica d'italia. /l Medioevo, Firenze, 1963; PH. JONES, "La storia economica. Dalla caduta dell'Imperio romano al secolo XIV" e "Economia e società nell'Italia medievale: la leggenda della borghesia", rispettivamente in Storia d'Italia, vol. II, e relativi Annali, vol. I, torino, 1974 e 1978; A. SCHAUBE, Storia del commercio dei popoli latini del Mediterraneo sino alla fine delle Crociate, trad. ital. Torino, 1915; F. CARLI, // mercato nell'alto Medioevo e // mercato nell'età comune, Padiva, 1934 e 1936; R.S. LOPEZ, I.W. RAYMOND, Medieval Trade in the Mediterranean World. Illustrative Documents. Translated with Introductions and Noptes, New York, 1955; P. TOUBERT, "Il sistema curtense: la produzione e lo scambio interno in Italia nei secoli VIII, IX e X", in Annali della Storia d'Italia, vol. VI,. Torino, 1983, pp. 3-63; R.S. LOPEZ, La rivoluzione commerciale del Medioevo, trad. it. Torino, 1975; A.O. 
CITARELLA, // commercio di Amalfi nell'alto Medioevo, Salerno, 1977; B. FIGLIUOLO, amalfi e il Levante nel Medioevo, in VV.AA. I Comuni italiani nel Regno crociato di Gerusalemme, Genova, 1986; F.C. LANE, Storia di Venezia, trad. it. Torino, 1978; G. LUZZATTO, Storia economica di Venezia dall'XI al XVI secolo, Venezia, 1961; D.S.H. ABULAFIA, The two Italies: economic relations betwen the Norman Kingdom of Sicily and the northen Communes, Cambridge, 1977; AA.VV.Le Italie del tardo Medioevo, Pisa, 1990 (relaz. di ;. Tangheroni, B. Dini, L. Travaiani, A. Cortonesi, G. Pinto, U. Tucci, L. Ciappa Mauri, H. Bresc); F. MELIS, I trasporti e le comunicazioni nel Medioevo, Firenze, 1984; TH. SZABO, Comuni e politica stradale in Toscana $e$ in Italia nel Medioevo, Bologna, 1992; G. CHERUBINI, L'Italia rurale del basso Medioevo, Roma-Bari, 1985; e Le città italiane dell'età di Dante, Pisa, 1991. 